\title{
Intelligent PID Controllers
}

\author{
Michel Fliess and Cédric Join
}

\begin{abstract}
Intelligent PID controllers, or i-PID controllers, are PID controllers where the unknown parts of the plant, which might be highly nonlinear and/or time-varying, are taken into account without any modeling procedure. Our main tool is an online numerical differentiator, which is based on easily implementable fast estimation and identification techniques. Several numerical experiments demonstrate the efficiency of our method when compared to more classic PID regulators.
\end{abstract}

Key words - PID control, intelligent PID controllers, modelfree control, fast estimation techniques, numerical differentiation, linear systems, nonlinear systems, flatness-based control, delay systems.

\section{INTRODUCTION}

Recent model-free control strategies have been proposed in [16] and [15]. They are based on new techniques for fast identification and estimation (see [17] and the references therein) and they have been shown to be quite efficient not only via academic examples in [15], [16] but also thanks to concrete case-studies $^{1}$ in [23], [27], [48].

This setting is based for a SISO system on the local description

$$
y^{(n)}=F+\alpha u
$$

where $\alpha$ is some given constant. The function $F=y^{(n)}-$ $\alpha u$, which is obtained via an estimation of the $n^{\text {th }}$-order derivative of the output signal, carries the whole information on the process we want to control: it might include severe nonlinearities as well as complex time-varying phenomena, like frictions. In all the examples we have investigated until now, we took $n=1$ or $n=2$. If $n=2$, we obtained the desired behavior via the following intelligent PID controller ${ }^{2}$, or $i$-PID controller,

$$
u=-\frac{F}{\alpha}+\frac{\ddot{y}^{*}}{\alpha}+K_{P} e+K_{I} \int e+K_{D} \dot{e}
$$

where $y^{*}$ is a reference output trajectory, and $e=y-y^{*}$. The aim of this communication is to show the superiority of those i-PID regulators with respect to more classic approaches of PID control.

Our paper is organized as follows. Sect. II is devoted to a short review of model-free control and gives the definition of

M. Fliess is with INRIA-ALIEN and LIX (CNRS, UMR 7161), École polytechnique, 91128 Palaiseau, France. Michel.FLiess@polytechnique.edu

C. Join is with INRIA-ALIEN and CRAN (CNRS, UMR 7039), Nancy-Université, BP 239, 54506 Vandœuvre-lès-Nancy, France. cedric.joinecran.uhp-nancy. fr

${ }^{1}$ Some industrial implementations, which will be discussed elsewhere, are already being developed.

${ }^{2}$ This terminology has already been used by other authors (see, e.g., [4]), but with a quite different meaning.
i-PID controllers. It contains moreover the basic principles of our numerical differentiators. The three examples of Sect. III, with numerous convincing computer simulations, are

- a stable linear system,

- an unstable nonlinear system,

- a spring with unknown damping, friction and nonlinearity.

The conclusion in Sect. IV is stressing the benefits of our viewpoint for industrial control applications, and lists some open questions.

Remark 1: We are perfectly aware that such a comparison with existing techniques may be objected on the basis of the huge literature on PID controllers since the founding papers by Ziegler \& Nichols [52], Cohen \& Coon [8], and Kessler [28] (see, e.g., [1], [2], [3], [5], [6], [7], [9], [10], [11], [12], [21], [22], [24], [26], [29], [30], [31], [32], [33], [34], [36], [38], [40], [41], [42], [43], [45], [47], [49], [51] and the references therein). It might always be argued that some already existing methodologies were ignored and/or misused. Only time and the combined effort of many control engineers will be able to establish our thesis on a completely firm basis.

\section{I-PID CONTROLLERS}

\section{A. Model-free setting ${ }^{3}$}

1) General principles: Take a finite-dimensional SISO system

$$
E\left(t, y, \dot{y}, \ldots, y^{(\iota)}, u, \dot{u}, \ldots, u^{(\kappa)}\right)=0
$$

which is linear or not, where $E$ is a sufficiently smooth function of its arguments. Assume that for some integer $n$, $0<n \leq \iota, \frac{\partial E}{\partial y^{(n)}} \not \equiv 0$. The implicit function theorem yields then locally

$$
y^{(n)}=\mathfrak{E}\left(t, y, \dot{y}, \ldots, y^{(n-1)}, y^{(n+1)}, \ldots, y^{(\iota)}, u, \dot{u}, \ldots, u^{(\kappa)}\right)
$$

By setting $\mathfrak{E}=F+\alpha u$, we obtain Eq. (1) where

- $\alpha \in \mathbb{R}$ is a non-physical constant parameter, which is chosen by the engineer in such a way that $F$ and $\alpha u$ are of the same magnitude,

- $F$ is determined thanks to the knowledge of $u, \alpha$, and of the estimate of $y^{(n)}$.

Remark 2: A system might only be partially unknown as in Sect. III-C. It is straightforward to adapt the previous method to this case.

\footnotetext{
${ }^{3}$ See [16] and [15] for more details.
} 
2) Numerical differentiation ${ }^{4}$ : Start with a polynomial time function $x_{N}(t)=\sum_{\nu=0}^{N} x^{(\nu)}(0) \frac{t^{\nu}}{\nu !} \in \mathbb{R}[t], t \geq 0$, of degree $N$. The usual notations of operational calculus (see, e.g., [50]) yield $X_{N}(s)=\sum_{\nu=0}^{N} \frac{x^{(\nu)}(0)}{s^{\nu+1}}$. Multiply both sides by positive powers of the algebraic derivative $\frac{d}{d s}$. The quantities $x^{(\nu)}(0), \nu=0,1, \ldots, N$, which are linearly identifiable, satisfy the following triangular system of linear equations:

$$
\frac{d^{\alpha} s^{N+1} X_{N}}{d s^{\alpha}}=\frac{d^{\alpha}}{d s^{\alpha}}\left(\sum_{\nu=0}^{N} x^{(\nu)}(0) s^{N-\nu}\right) \quad 0 \leq \alpha \leq N-1
$$

Multiplying both sides of Eq. (3) by $s^{-\bar{N}}, \bar{N}>N$, permits to get rid of time derivatives, i.e., of $s^{\mu} \frac{d^{\iota} X_{N}}{d s^{\iota}}, \mu=1, \ldots, N$, $0 \leq \iota \leq N$.

Consider now an analytic time function, defined by the power series $x(t)=\sum_{\nu=0}^{\infty} x^{(\nu)}(0) \frac{t^{\nu}}{\nu !}$, which is assumed to be convergent around $t=0$. Approximate $x(t)$ by the truncated Taylor expansion $x_{N}(t)=\sum_{\nu=0}^{N} x^{(\nu)}(0) \frac{t^{\nu}}{\nu !}$ of order $N$. Good estimates of the derivatives are obtained by the same calculations as above.

3) Noise removal: Corrupting noises are viewed as highly fluctuating phenomena, which are attenuated via low pass filters (see [13] for more details). Such filters may be obtained for instance by multiplying both sides of Eq. (3) by $s^{-\bar{N}}$, where $\bar{N}>0$ is large enough.

\section{B. Controllers}

If $n=2$ in Eq. (1), the desired behavior is obtained via the intelligent PID controller, or i-PID controller, (2) where

- $K_{P}, K_{I}, K_{D}$ are the tuning parameters,

- $y^{*}$ is a reference output trajectory,

- $e=y-y^{*}$ is the output tracking error.

Remark 3: If $n=1$ in Eq. (1), replace Eq. (2) by the intelligent PI controller, or $i$-PI controller,

$$
u=-\frac{F}{\alpha}+\frac{\dot{y}^{*}}{\alpha}+K_{P} e+K_{I} \int e
$$

Remark 4: We were never obliged until now to choose $n \gtreqless 2$ in Eq. (1). If nevertheless this happens we would easily extend the generalized proportional-integral controllers, or GPI controllers, of [19] to intelligent generalized proportional-integral controllers, or $i$-GPI controllers.

Let us emphasize the following differences with respect to more classic approaches of PID controllers:

- No identification procedure is needed since the whole structural information is contained in the term $F$ which is canceled. We are able to control high dimensional and/or strongly nonlinear systems without any complex and time-consuming parameter tuning.

- The flatness-based-like output reference trajectory (see, e.g., [18], [44]) is much more flexible than the step trajectory between two setpoints which still is quite common in industry. It permits to a large extent to avoid overshoots and undershoots.

\footnotetext{
${ }^{4}$ See [35] for the most recent and powerful advances. The reference [17]
} contains a quite extensive bibliography on this subject.

\section{COMPUTER SIMULATIONS AND COMPARISONS}

All computer simulations are performed with a corrupting additive white Gaussian noise, which is zero-mean and with variance 0.01 . With classic PID regulators standard low-pass filters are utilized whereas robustness with respect to noises for i-PID controllers is ensured via the principles of Sect. II-A.3.

\section{A. A stable linear system}

The transfer function

$$
\frac{(s+2)^{2}}{(s+1)^{3}}
$$

defines a stable linear system.

1) A classic PID controller: We follow Dindeleux [11] and apply Broïda's method by approximating the inputoutput behavior (5) by the delay system

$$
\frac{K e^{-\tau s}}{(T s+1)}
$$

The parameters $K, T, \tau$ are obtained via graphical methods: $K=4, T=2.018, \tau=0.2424$. It yields the following PID coefficients: $K_{P}=\frac{100(0.4 \tau+T)}{120 K \tau}=1.8181, K_{I}=\frac{1}{1.33 K \tau}=$ $0.7754, K_{D}=\frac{0.35 T}{K}=0.1766$.

2) The $i$-PI controller: The very simple model $\dot{y}=F+u$ is used. Write $[\bullet]_{e}$ the estimate of a given quantity. Introduce the i-PI controller

$$
u=-[F]_{e}+\dot{y}^{\star}+\operatorname{PI}(e)
$$

where

- $[F]_{e}=[\dot{y}]_{e}-u$,

- $y^{\star}$ is a reference trajectory,

- $e=y-y^{\star}$,

- $\operatorname{PI}(e)$ is a standard PI controller.

3) Simulation results: Fig. 1 shows that our i-PI controller behaves slightly better than the classic PID regulator. The situation is much more revealing if we assume that

- our system is changing with time,

- a fault is occurring.

The system poles are no more located at 1 but at 1.5, i.e., its transfer function becomes

$$
\frac{(s+2)^{2}}{(s+1.5)^{3}}
$$

Fig. 2 demonstrates that the difference between our i-PI controller becomes quite important with the classic PID regulator if we do not perform again the graphical identification techniques.

The actuator failure in Fig. 3 is a $50 \%$ power loss. Here again our i-PI controller behaves much better than the standard PID controller.

\section{B. An unstable nonlinear system}

Consider the unstable nonlinear system

$$
\dot{y}-y=u^{3}
$$




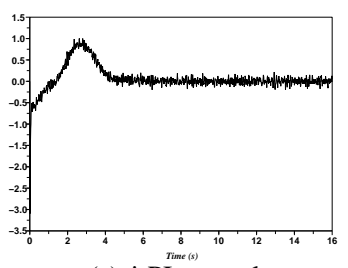

(a) i-PI control

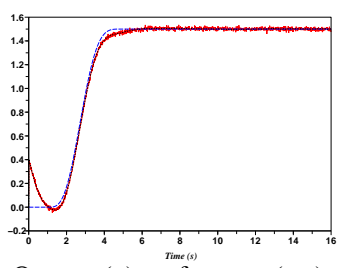

(b) Output (-); reference (- -); denoised output (. .)

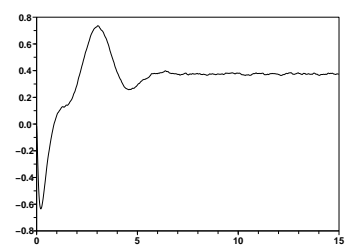

(c) PID control

Fig. 1. Stable linear system

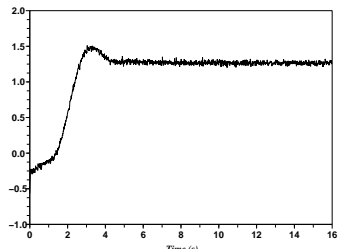

(a) i-PI control

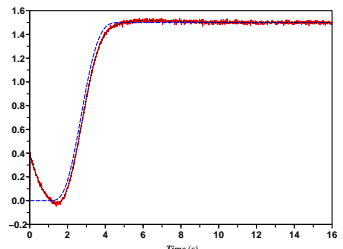

(b) Output (-); reference (- -); denoised output (. .)

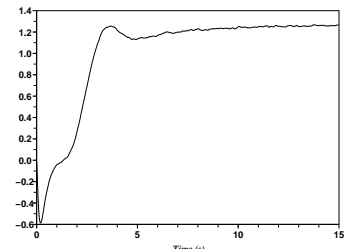

(c) PID Control

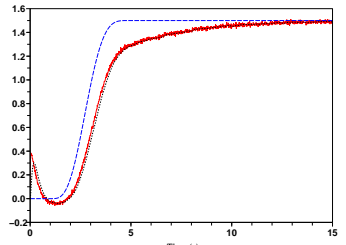

(d) Output (-); reference (- -); denoised output (. .)

Fig. 2. Modified stable linear system

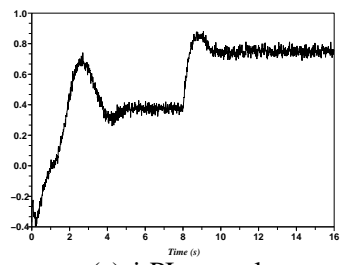

(a) i-PI control

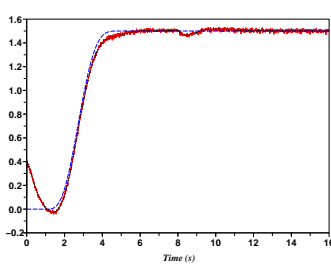

(b) Output (-); reference (- -); denoised output (. .)

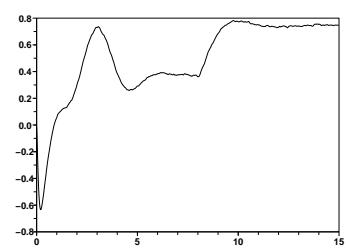

(c) PID control

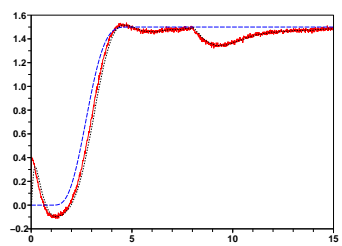

(d) Output (-); reference (- -); denoised output (. .)

Fig. 3. Stable linear system with actuator failure

1) A classic PID controller: As in Sect. III-A.1, we approximate Eq. (6) via the delay system

$$
\frac{K e^{-\tau s}}{s}
$$

The parameters $K, \tau$ are again obtained via a graphical method around the operating point $u=1, y=1.5$ : $K=2$, $\tau=0.2$. It yields according to [11] the PID coefficients: $K_{p}=\frac{100}{110 K \tau}=2.2727, K_{i}=\frac{1}{6.66 K \tau^{2}}=1.8769$ and $K_{d}=\frac{0.35}{K}=0.175$.

Remark 5: The quality of the above parameters strongly depends on the accuracy of the identification by Eq. (7). This identification here is quite "subtle", i.e., not very natural.

2) The i-PI controller: The coefficients $K_{P}$ and $K_{I}$ in Eq. (4) are selected in such that -2 is the double pole of the closed loop dynamics.

3) Simulation results: Fig. 4 shows the poor performances of the classic PID control with steps as reference trajectories. Note that for the numerical simulations we utilized for the PID controller, as often in industry, only the derivative of the output variable $y$ :

$$
u=K_{P}\left([y]_{e}-y^{\star}\right)+K_{I} \int\left(y-y^{\star}\right)+K_{D}[\dot{y}]_{e}
$$

Replace, following the principles of flatness-based control, the step reference trajectories by suitable smooth trajectories, which are defined here via Bézier polynomials. Even if the performance of the classic PID regulator increases, Figs. 5 and 6 demonstrate that the i-PI controller behaves much better.

4) Anti-windup: Set, for instance, $-2 \leq u \leq 0.4$. According to Fig. 7 we obtain second-rate performances without anti-windup (see, e.g., [25]). With an anti-windup on the classical part of the i-PI controller, Fig. 8 shows that the reference trajectory should be modified according to the principles of flatness-based control.

\section{An example of a partially known system}

Consider the following spring

$$
m \ddot{y}=-\mathcal{K}(y)+\mathcal{F}(\dot{y})-d \dot{y}+F_{\text {ext }}
$$

where $y$ is the position of a point mass $m, F_{\text {ext }}=u$ is the control, $d$ is the damping, $\mathcal{K}(y)=k_{1} y+k_{3} y^{3}$ contains a cubic Duffing nonlinearity, $\mathcal{F}(\dot{y})$ is the friction. The mass $m=0.5$ is perfectly known. The error on the coefficient $k_{1}=5$ is $40 \%$ since we are utilizing the value $\hat{k}_{1}=3$ in the simulations. The true values of the coefficients $d$ and $k_{3}$, which are unknown, are 1 and 2 in the simulations. 


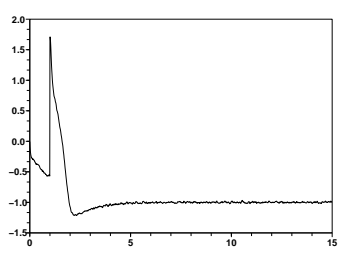

(a) PID control

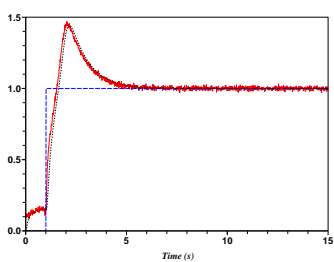

(b) Output (-); reference (- -); denoised output (. .)

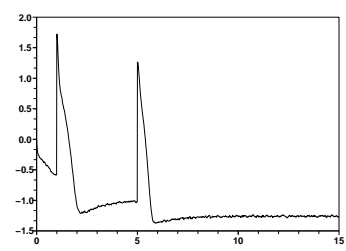

(c) PID control

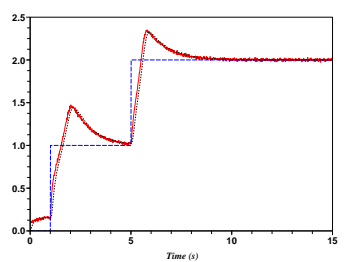

(d) Output (-); reference (- -); denoised output (. .)

Fig. 4. PID control for the unstable nonlinear system with steps as reference trajectory

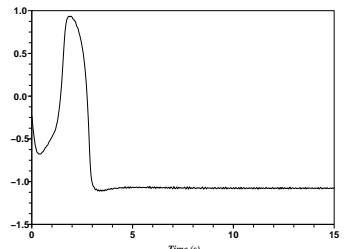

(a) i-PI control

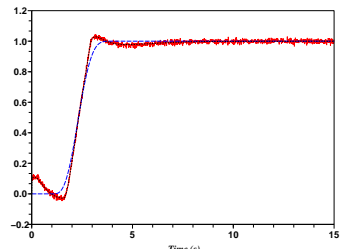

(b) Output (-); reference (- -); denoised output (. .)

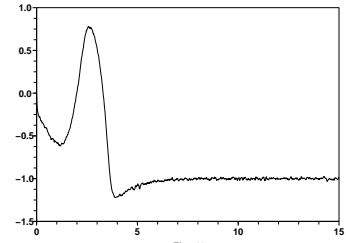

(c) PID control

Fig. 5. Unstable nonlinear system with a smooth reference trajectory

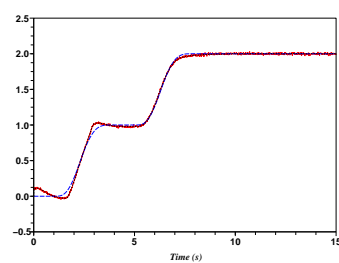

(b) Output (-); reference (- -); denoised output (. .)

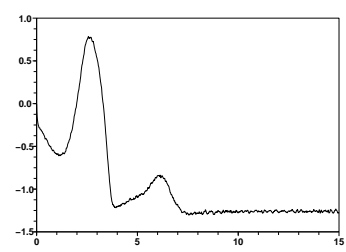

(c) PID control

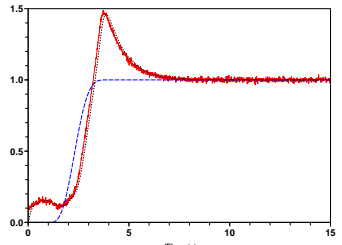

(d) Output (-); reference (- -); denoised output (. .)

Fig. 6. Unstable nonlinear system with another smooth reference trajectory

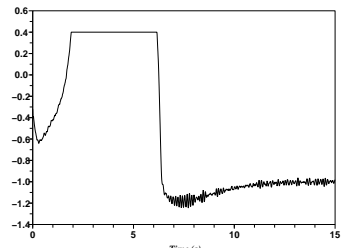

(a) Contro

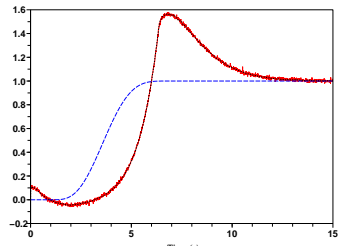

(b) Output (-); reference (- -); denoised output (.)

Fig. 7. Unstable nonlinear system: saturated control without anti-windup

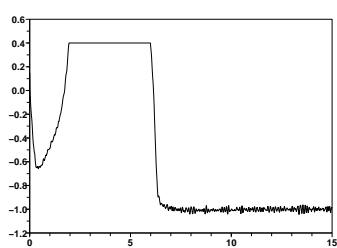

(a) Control

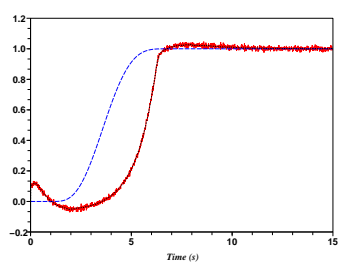

(b) Output (-); reference (- -); denoised output (. )

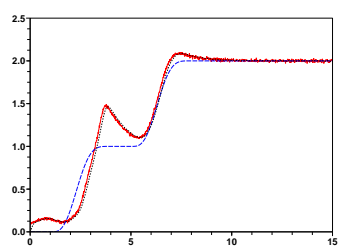

(d) Output (-); reference (- -); denoised output (. .) 
Choose for the friction ${ }^{5}$ in the numerical simulations the classic Tustin model [46]: Fig. 9-(a) shows a quite "brutal" behavior when the sign of the speed is changing.

1) A classic PID controller: We design a PID controller with respect to the partially known model $m \ddot{y}=-\hat{k}_{1} y+u$. Its coefficients are selected such that all closed loop poles are equal to $-3: K_{p}=-\hat{k}_{1}+27 m, K_{i}=-27 m, K_{d}=9 m$.

2) The $i$-PID controller: Select a reference trajectory $y^{\star}$. Set $u^{\star}=m \ddot{y}^{\star}+\hat{k}_{1} y^{\star}$. The designed control is

$$
F_{\text {ext }}=u=u^{\star}-[\mathcal{G}]_{e}+\operatorname{PID}(e)
$$

where

- $\mathcal{G}=\mathcal{F}(\dot{y})-\left(k_{1}-\hat{k}_{1}\right) y-k_{3} y^{3}-d \dot{y}$, which is bringing together all the complex and poorly known phenomena (damping, friction, nonlinearity), is estimated via

$$
[\mathcal{G}]_{e}=m[\ddot{y}]_{e}+\hat{k}[y]_{e}-F_{\text {ext }}
$$

which is deduced from Eq. (8).

- The i-PID controller $\operatorname{PID}(e), e=y-y^{\star}$, is chosen such that -3 is the only pole.

3) Simulation results: Fig. 9-(c,d) demonstrate excellent results for the control (9), when compared to those in

- Fig. 9-(e,f) with a flatness-based nominal trajectory and a classic PID controller, but without taking into account the unknown effects,

- Fig. 9-(g,h) with a classic PID controller, but without a flatness-based nominal trajectory and without taking into account the unknown effects.

When the friction is becoming more important, the tracking performances without estimating $\mathcal{G}$ are deteriorating whereas those of the i-PID controller (9) are unaltered.

\section{CONCLUSION}

The first two examples seem to show that delay systems defined by transfer functions of the form $T(s) e^{-L s}, T \in$ $\mathbb{R}(s), L \geq 0$, which play since Ziegler \& Nichols [52] such a fundamental role in the parameter tuning of PID controllers, might loose their importance. See [20] for a general study of this class of systems.

Consider the following problems with classic PID regulators:

- How to ensure good performances with disparate plants without having to tune again and again the PID parameters?

- How to guarantee a suitable adaptation when the plant is changing with time?

Our intelligent PID controllers offer a most efficient answer to those crucial points for any industrial application, which is moreover easily implementable: $F$ in Eq. (1) is estimated online.

The first example in Sect. III-A demonstrates that the familiar mathematical robustness criteria are becoming pointless here. It should be clear that the robustness of our i-PID

\footnotetext{
${ }^{5}$ Tribology is a most difficult domain which is still attracting a lot of studies (see, e.g., [37], [39], and the references therein). Let us stress here that we do not need here any modeling of frictions.
}

controllers depends on practical implementation issues, like the quality of the sensors, the magnitude of the corrupting noises, the sampling period, and the computer capability.

A more detailed presentation will appear in [14], where some important questions, like multivariable systems, and non-minimum phase systems, will also be examined.

Acknowledgements. The authors would like to thank Dr. O. Gibaru (INRIA-ALIEN - École des Arts \& Métiers ParisTech, Lille) for some helpful discussions.

\section{REFERENCES}

[1] Alfaro, V.M., PID controllers'fragility, ISA Trans., vol. 46, 2007, 555-559.

[2] Ang, K.H., Chong, G., Li, Y., PID control system analysis, design, and technology, IEEE Trans. Control Systems Techn., vol. $13,2005,559-576$.

[3] Aström, K.J., Hägglund, T., Advanced PID Control, Instrument Soc. Amer., 2006.

[4] Aström, K.J., Hang, C.C., Persson, P., Ho, W.K., Towards intelligent PID control, Automatica, vol. 28, 1992, 1-9.

[5] Besançon-Voda, A., Gentil, S., Régulateurs PID analogiques et numériques, Techniques de l'Ingénieur, R7416, 1999.

[6] Chen, J., Huang, T.-C., Applying neural networks to online updated PID controllers for nonlinear process control, $J$. Process Control, vol. 14, 2004, 211-230.

[7] Choi, Y., Chung, W.K., PID Trajectory Tracking Control for Mechanical Systems, Springer, 2004.

[8] Cohen, G.H., Coon, G.A., Theoretical consideration of retarded control, Trans. Amer. Soc. Mech. Eng., vol. 75, 1953, 827-834.

[9] Datta, A., Ho, M.T., Bhattacharyya, S.P., Structure and Synthesis of PID Controllers, Springer, 2000.

[10] Dieulesaint, E., Royer D., Automatique appliquée - t. 1 : les procédés physiques de transformation, Masson, 1987.

[11] Dindeleux, D., Technique de la regulation industrielle, Eyrolles, 1981.

[12] Flaus, J.-M., La régulation industrielle, Hermès, 1994.

[13] Fliess, M., Analyse non standard du bruit, C.R. Acad. Sci. Paris Ser. I, vol. 342, 2006, 797-802.

[14] Fliess, M., Join, C., Commande sans modèle et commande à modèle restreint, $e-S T A$, vol. 5,2008 . Soon available at http://hal.inria.fr.

[15] Fliess, M., Join, C., Mboup, M., Sira-Ramírez, H., Vers une commande multivariable sans modèle, Actes Conf. Francoph. Automat. (CIFA-2006), Bordeaux, 2006. Available at http://hal.inria.fr/inria-00001139/en/.

[16] Fliess, M., Join, C., Sira-Ramírez, H., Complex continuous nonlinear systems: their black box identification and their control, Proc. $14^{\text {th }}$ IFAC Symp. System Identif. (SYSID-2006), Newcastle, Australia, 2006. Available at http://hal.inria.fr/inria-00000824/en/.

[17] Fliess, M., Join, C., Sira-Ramírez, H., Nonlinear estimation is easy, Int. J. Modelling Identification Control, vol. 3, 2008. Available at http://hal.inria.fr/inria-00158855/en/.

[18] Fliess, M., Lévine, L., Martin, P., Rouchon, P., Flatness and defect of non-linear systems: introductory theory and examples, Int. J. Control, vol. 61, 1995, 1327-1361.

[19] Fliess, M., Marquez, R., Delaleau, E., Sira-Ramírez, H., Correcteurs proportionnels-intégraux généralisés, ESAIM Control Optim. Calc. Variat., vol. 7, 2002, 23-41.

[20] Fliess, M., Marquez, R., Mounier, H., An extension of predictive control, PID regulators and Smith predictors to some delay systems, Int. J. Control, vol. 75, 2002, 728-743.

[21] Föllinger, O., Regelungstechnik - Einführung in die Methoden und ihre Anwendung, Hüthig Verlag, 1994. 


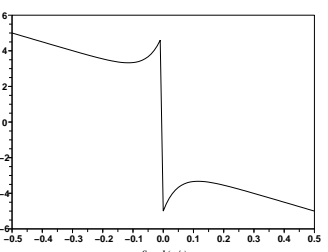

(a) Friction function

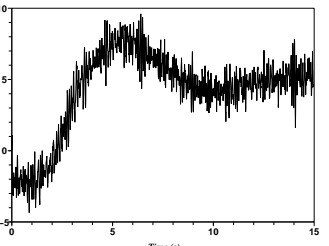

(e) PID control

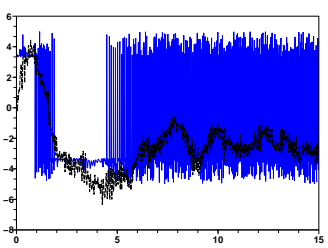

(b) Friction, $\mathcal{F}(\dot{y}),(-)$; unknown input estimation, $[\mathcal{G}(\dot{y})]_{e}$, filtered (-)

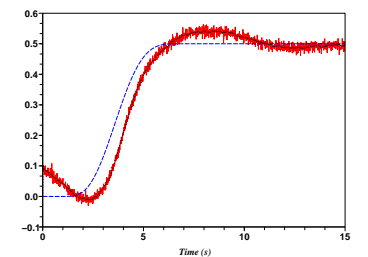

(f) PID control: output (-); reference (- -); denoised output (. .)

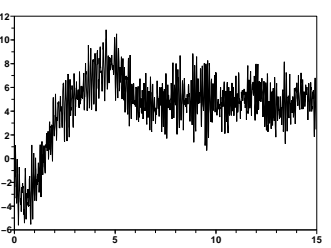

(c) i-PID control

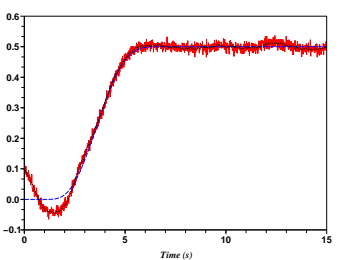

(d) i-PID control: output (-); reference (- -); denoised output (. .)

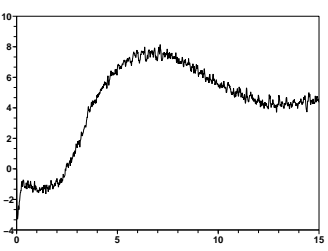

(g) PID control with more important friction

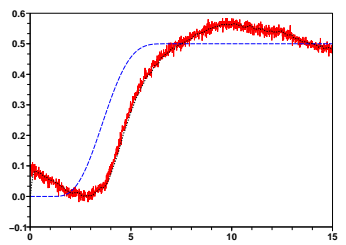

h) PID control with more important friction: output (-); reference (- -); denoised output (. .)

Fig. 9. Spring with unknown nonlinearity, friction, and damping

[22] Franklin, G.F., Powell, J.D., Emami-Naeini, A., Feedback Control of Dynamic Systems, ${ }^{\text {th }}$ ed., Prentice Hall, 2002.

[23] Gédouin, P.-A., Join, C., Delaleau, E., Bourgeot, J.-M., Chirani, S.A., Calloch, S., Model-free control of shape memory alloys antagonistic actuators, Proc. $17^{\text {th }}$ IFAC World Congress (WIFAC-2008), Seoul, 2008. Available at http://hal.inria.fr/inria-00261891/en/.

[24] Giöngy, I.J., Clarke, D.W., On the automatic tuning and adaptation of PID controllers, Control Eng. Pract., vol. 14, 2006, 149-163.

[25] Hippe, P., Windup in Control - Its Effects and Their Prevention, Springer, 2006.

[26] Johnson, M.A., Moradi, M.H., Crowe, J., Eds., PID Control: New Identification and Design Methods, Springer, 2005.

[27] Join, C., Masse, J., Fliess, M., Étude préliminaire d'une commande sans modèle pour papillon de moteur, J. europ. syst. automat., vol. 42, 2008, 337-354.

[28] Kessler, C., Das symmetrische Optimum, Regelungstechnik, vol. 6, 1958, 395-400 \& 432-436.

[29] de Larminat, P., Automatique appliquée, Hermès, 2007.

[30] Lequesne, D., Régulation P.I.D., Hermès, 2006.

[31] Lequin, O., Gevers, M., Mossberg, M., Bosmans, E., Triest, L., Iterative feedback tuning of PID parameters: comparison with classical tuning rules, Control Eng. Pract., vol. 11, 2003, 1023-1033.

[32] Li, Y., Ang, K.H., Chong, G.C.Y., PID control system analysis and design, IEEE Control Systems Magaz., vol. 26, 2006, 3241.

[33] Li, T., Xiong, J., Zhang, R., Tan, Q., Xu, R., Hardware implementation of fuzzy PID controllers, Fuzzy Optim. Decis. Making, vol. 5, 2006, 113-122.

[34] Longchamp, R., Commande numérique des systèmes dynamiques, $2^{e}$ éd., Presses polytech. universit. romandes, 2006.

[35] Mboup, M., Join, C., Fliess, M., A revised look at numerical differentiation with an application to nonlinear feedback control, Proc. $15^{\text {th }}$ Mediterrean Conf. Control Automation (MED-2007), Athens, 2007. Available at http://hal.inria.fr/inria-00142588/en/.

[36] Morari, M., Zafiriou, E., Robust Process Control, Prentice Hall, 1989.

[37] Nuninger, W., Perruquetti, W., Richard, J.-P., Bilan et enjeux des modèles de frottements: tribologie et contrôle au service de la sécurité des transports, Actes $5^{e}$ Journées Europ. Freinage (JEF-2006), Lille, 2006. Available at http://hal.inria.fr/inria-00192425/en/.

[38] O'Dwyer, A., Handbook of PI and PID Controller Tuning Rules, $2^{\text {nd }}$ ed., Imperial College Press, 2006.

[39] Olsson, H., Aström, K. J., Canudas de Wit, C., Gäfvert, M., Lischinsky, P., Friction models and friction compensation, Europ. J. Control, vol. 4, 1998, 176-195.

[40] Pigeron, B., Mullot, H., Chaix, A., Félix, L., Aubert, Y., Boucles de régulation, $4^{e}$ éd., BHALY Autoédition, 2005.

[41] Reznik, L., Ghanayem, O., Bourmistrov, A., PID plus fuzzy controller structures as a design base for industrial applications, Eng. Appl. Artif. Intelligence, vol. 13, 2000, 419-430.

[42] Shinskey, F.G., Process Control Systems - Application, Design, and Tuning, $4^{\text {th }}$ ed., McGraw-Hill, 1996.

[43] Silva, G.J., Datta, A., Bhattacharyya, S.P., On the stability and controller robustness of some popular PID tuning rules, IEEE Trans. Automat. Control, vol. 48, 2003, 1638-1641

[44] Sira-Ramírez, H., Agrawal, S., Differentially Flat Systems, Marcel Dekker, 2004.

[45] Skogestad, S., Simple analytic rules for model reduction and PID controller tuning, J. Process Control, vol. 13, 2003, 291$309 \&$ vol. $14,2004,465$.

[46] Tustin, A., The effect of backlash and speed-dependent friction on the stability of closed-cycle control systems, J. Instit. Electrical Eng., vol. 7, 1947, 699-706.

[47] Vilanova, R., IMC based robust PID design: Tuning guidelines and automatic tuning, J. Process Control, vol. 18, 2008, 61-70.

[48] Villagra, J., d'Andréa-Novel, B., Choi, S., Fliess, M., Mounier, H., Robust stop-and-go control strategy: an algebraic approach for nonlinear estimation and control, Int. J. Vehicle Autonomous Systems, to appear. Soon available at http: //hal.inria.fr.

[49] Visioli, A., Practical PID Control, Springer, 2006.

[50] Yosida, K., Operational Calculus: A Theory of Hyperfunctions, Springer, 1984 (translated from the Japanese).

[51] Yu, C.C., Autotuning of PID Controllers, Springer, 1999.

[52] Ziegler, J.G., Nichols, N.B., Optimum settings for automatic controllers, Trans. Amer. Soc. Mech. Eng., vol. 64, 1942, 759768. 